\title{
VERTICAL INTEGRATION ON INDUSTRIAL EXAMPLES
}

\author{
Andreas Dedinak, Christian Wögerer, Helmut Haslinger, Peter Hadinger \\ ARC Seibersdorf research $\mathrm{GmbH}$ \\ andreas.dedinak@arcs.ac.at,christian.woegerer@arcs.ac.at \\ helmut.haslinger@arcs.ac.at,peter.hadinger@arcs.ac.at
}

AUSTRIA

\begin{abstract}
"Vertical integration" is an often used headline in the closed communication from office to machine level The paper will give an overview of state-of-the-art, problems as well as opportunities of concept. Apart from a more general problem description, the paper also reports on results exemplarily in 3 industrial projects achieved by ARC Seibersdorf Research GmbH. The first example covers industrial measurement and testing automation - the most modern test stand for heat meter calibration set up for the PTB laboratories in Berlin. The second one shows the development of a full-automated casting plant for magnesium implementing the safety system. The last one is a highspeed coin sorting system for more than 1000 coin types consisting of control, visualisation, image processing and database.
\end{abstract}

\section{INTRODUCTION}

Integration of the main business levels of a production or process orientated enterprise reduces costs, leads to higher flexibility - ending up in prompt time to market and increased quality. As this vertical integration will play a major role in the automation business within the upcoming years, this paper will give an overview over this topic.

First one addresses the issue of the motivation for a structured networking of different business levels. The stimulus is the existing situation which nowadays often leads to problems in not meeting the right delivery date, not meeting the according quality, not being competitive, not being flexible, etc. These problems often arise in historical grown companies, where the individual business levels build their own islands, their own solutions for their own problems with lacking interprocess communication. This situation makes production enlargement difficult, implementation of new technologies expensive, inhibits establishment of standards and makes processes and data difficult to be understood. Moreover, planned and actual status are hard to compare.

\subsection{Upcoming Business Trends}

The above described lack of in-house business networking is further increased by todays and tomorrows demands on modern business units: 
- Product life cycles shortened, even technological cycles shortened

- Orders to stock replaced by short orders to delivery

- Shift form in-house production to integration of sub suppliers

- Decentralised stock and service

- High pricing pressure

- Shift form manual process integration to integrated processes

- Shift from product supplier to system supplier

- Production processes control multiple enterprises

- E-commerce and quality management systems additionally produce enormous quantities of data and require the introduction of a data management throughout the company

These demands can only be met by the horizontal and vertical integration of business units within the company, and exceeding the network to suppliers and even customers.

\subsection{The Business Pyramid}

When speaking of vertical integration of business levels, the main levels in a production or process-orientated enterprise should be defined:

- Enterprise Planning Level: Uppermost managing level, covers the enterprise management as well finance, logistic and human resources.

- Factory Management Level: covers production planning, production analysis, production optimisation and quality control.

- Production Management Level: covers production control and monitoring, production data concentration, production cell automation, SCADA systems, etc.

- Process Level: covers process control including sensors and actors for signal acquisition and control execution.

In near future the limits between the enterprise levels will become blurred (vertical integration) and all steps within a process chain will be linked (horizontal integration). As a final result, productivity will grow.

\section{COMMUNICATION AS THE KEY FACTOR}

Analyzing the necessary methods for integrating business islands vertically and horizontally reveals communication as the key factor. The data and communication pyramid has to represent the enterprise pyramid. A sweeping communication network from the sensor level to the managing level is evident, although the demands of such networks differ in individual levels. The requirements on field (production area) related communication networks (busses) are:

- availability very high, as production breakdowns are possible

- changes ins system configuration seldom

- distances possibly high $(>100 \mathrm{~m})$

- EMC pollution often high

- mechanical stress possibly high 
- temperature stress possibly high

- installation often by unskilled workers

- operation and service by process orientated personnel

In contrast, the request on office related communication networks (busses) are:

- availability medium, as "only" loss of working hours is possible

- changes ins system configuration frequently

- distances small $(>100 \mathrm{~m})$

- EMC pollution modest

- mechanical stress modest

- temperature stress modest (often air conditioned)

- installation by skilled personnel

- operation and service by network specialists

We will divide the communication methods in IT related technologies, in Data related technologies and in Field related technologies. Typical representatives of the IT technology are Ethernet, Industrial Ethernet and Wireless LAN (Local Area Network). Typical representatives of the Data related technology are Profibus, Interbus, F oundation F ieldbus, Modbus, Devicenet, etc. Typical representatives of the Field technology are ASI (Actor Sensor Interface bus), CAN (Controller Area Network), EIB (European Installation Bus), LON (Local Operating Network), etc. These networks often serve in their typical application environment and are linked together by converters or bridges connecting the individual levels of communication.

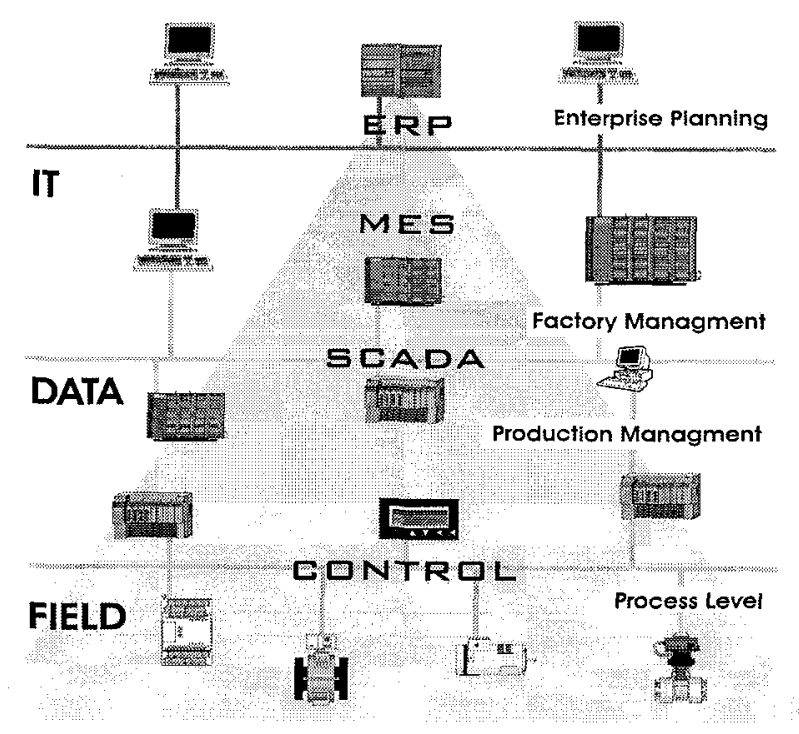

Figure 1 - The communication pyramid corresponds to the enterprise pyramid

This network of networks (busses) is frequently called the communication pyramid (Figure 1), because the members in the lowest communication hierarchy are numerical the largest group (sensors and actors), but have the smallest data rates. 
The members in the highest communication hierarchy are numerical the smallest group (servers), but have the highest data rates.

Nevertheless Ethernet and similar technologies penetrate in direction of field level. This results from a rising amount of data in the sensors and actors, as well as from decentralised operation of these devices.

Besides the hardware side of the communication network, the software technology is at least as important. The most important components in nonproprietary communication between software modules of different suppliers in production systems were established under the leadership of Microsoft Corp. and are based on their COM / DCOM ((Distributed) Component Object Model) system. Based on this object model with a clear defined access to the data and property interface of a binary software module, OPC (OLE for Process Control) established a standard in communication of automation systems in an unpredictable speed. All major suppliers of various automation products today support this standard.

Although OPC DA (Data Access) is the world wide standard, OPC further extends this standard to a variety of areas as batch processes (OPC Batch), historical data access (OPC HAD), data exchange between OPC servers (OPC DX) and last, but not least, OPC XML. With XML in general there will be a remedy for the big disadvantages of $\mathrm{OPC}$ - the inability to communicate to systems beyond the borders of the Microsoft platform. It remains to be seen whether OPC XML will overcome these ultimate barriers to an unlimited communication between automation products.

\section{MANUFACTURING EXECUTION SYSTEMS}

As workshop and enterprise level based automation software has been developed since decades without large-scale integration (also developed and operated by people of different educational backgrounds), the modern MES (Manufacturing Execution Systems) software seems to be turning to the missing link capable of bridging the gap of the above mentioned business levels. So far, MES is a loose, but powerful collection of software modules for tasks like production dispatching, resource management, production tracking, maintenance management, production execution, etc. Implementing the modules adapted to the respective business process should result in following benefits:

- Right data at the place of interest

- Real time data at the right moment

- Consistency in production databases

- Higher level of transparency

All these benefits should sum up to:

- INCREASED QUALITY

- SHORTER RESPONSE TIMES
- Higher level of reliability of decisions

- Observance of schedules

- Observance of quality rules

- Optimised Processes

- DECREASED COSTS

Nowadays software producer work on establishing clearly structured frameworks to increase efficiency and usability of these MES modules. Nevertheless 
today and in the near future these frameworks and their subsystems will work only in a proprietary manner; as yet standards for these MES frameworks are not implemented.

Combining today's opportunities of $\mathrm{e}$ - business with the potential of a fully networked production enterprise, the vision of a global market capable of overcoming physical distances and geographic locations by establishing enterprise structures and cooperation's as demanded by products or projects, is no longer a question of technical feasibility.

\section{INDUSTRIAL PROJECTS}

ARC Seibersdorf research has long time experience in mechanical and electrical automation of production and testing systems. Combining these skills with the knowledge of industrial networking, databases and interfaces for enterprise planning systems the ARC Seibersdorf research $\mathrm{GmbH}$ deals with the problems of integrating the workshop level to the management level since several years. Three exemplary projects will be described in short:

\subsection{Fully Automated Test Stand for Heat Meters with Integration of the Complete Test Administration}

ARC Seibersdorf research was put in charge to build on of the largest and most modern test stand for heat meters in Berlin. Customer was the well-known PTB (Physikalisch Technische Bundesanstalt).

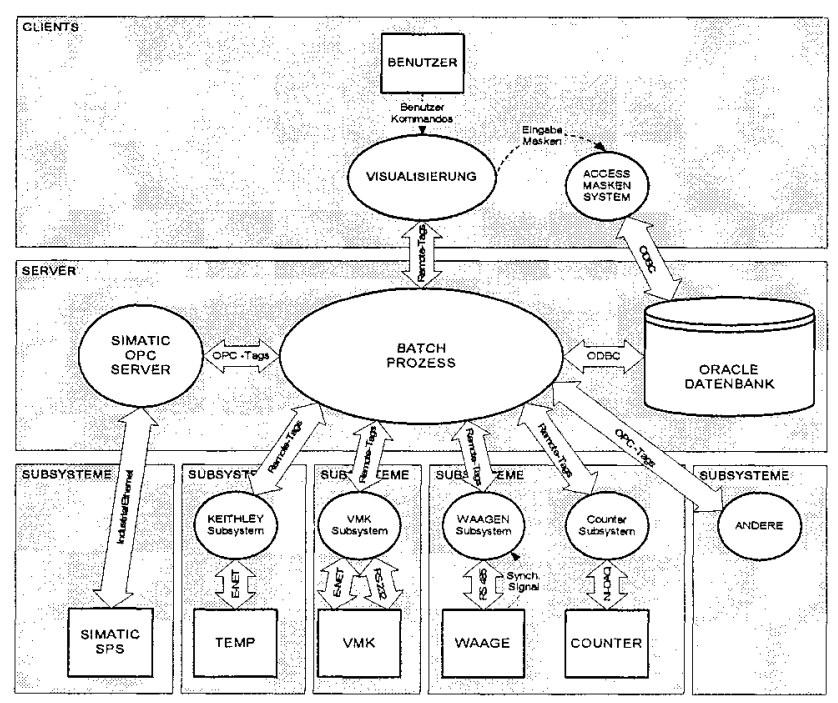

Figure 2 - Systems communication pyramid

In addition to build up the mechanic and measurement system challenging the frontiers of today's realizability, the test stand should be embedded in an overall test administration system. This starts up with the management of customers, test orders, 
devices under test and ends up in the management for test reports and quality management for the test stand with its subsystems itself (Figure 2). The essential step was the integration of the test stand management database (Oracle or MS SQL server) with its user front-end in the production database, organising the test routines itself and is responsible for the storage of real time trend data from the test process.

In these databases not only the test and test management data is stored, but also the complete setup and control parameters for the control system as well as the calibration data of the test stands gauges.

For exact repeatability of tests, all setup and control parameters of the complete facility can be reloaded to the subsystems automatically by calling a certain date or test order from the database. By additionally generating the necessary documents for quality control automatically, the customer not only owns one of the most modern test stands in measuring technology, but as in terms of data management.

\subsection{Control Technology for a continuous casting Plant}

Because of the familiar behaviour of magnesium alloys (strong exothermal reaction, explosion hazard) it was necessary to develop the whole processing chain starting from melting, alloying, casting, heat treatment and finally to extrusion of $\mathrm{Mg}$ profiles to provide a safe technology for the operators in $\mathrm{Mg}$ - direct chill foundries.

One key factor was the automation-, simulation- and control process. For a full automation it was necessary to adapt an existing casting plant, to develop a new launder system from the furnace to the die, new sensors (level, visualization) and to create a control - software witch is also able to control the plant and to log continuously various parameters of the casting process within maintenance via Internet.

\subsubsection{Control System}

During the casting process of magnesium the prevention of the oxidation reaction is the first goal. According to the potential explosive reaction of magnesium with the cooling water, no staff is allowed near the casting equipment during the process. At the same time it must be warranted that caster and material experts are able to optimize the process online at anytime.

The automation solution (Figure 3) bases on a high performance industrial controller (PLC) and a process visualisation and data management system (HMI Figure 4). The PLC controls the process parameters, all safety relevant limit values and the communication with the bus linked periphery equipment. The casting plant, the inert gas plant, the melting furnace and the casting plant for massels are individually controlled by an own PLC connected to the central controller. The periphery equipment of the casting plant for massels is connected via profibus with its controller.

\subsubsection{Visualisation}

Industrial Ethernet networks the HMI system to the control system. In this manner the casting process can be controlled from almost any distance and from any place of the world via Internet. Conditional on the integrated database it is furthermore possible to register process- and automation parameters online to save them for following analysis. This is basis for a further optimisation of the process. Naturally all data can be observed online by customer specified images, evaluated by various 
statistics and combined to trends or reports. The HMI system is able to allocate parameters by open interfaces like OPC to external evaluation programs.

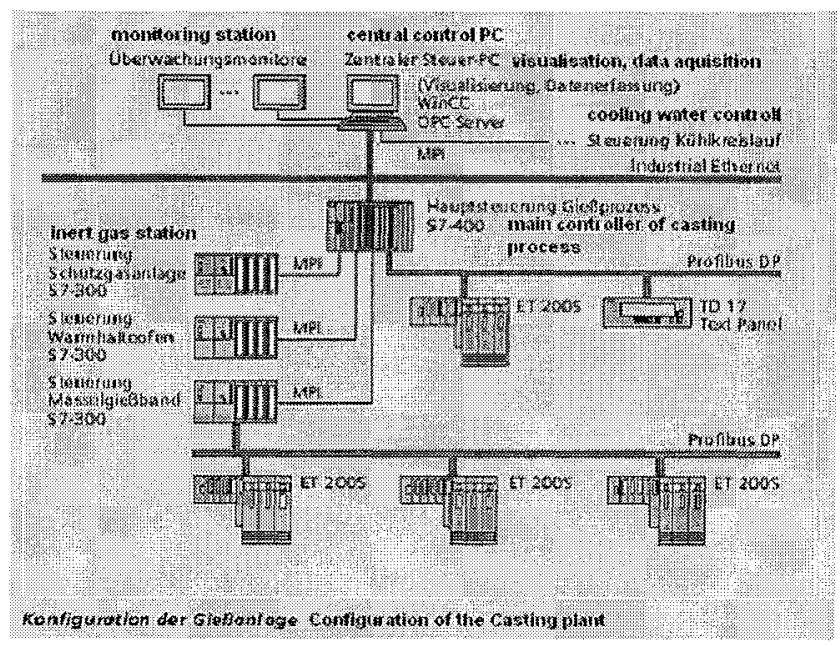

Figure 3 - Control Configuration of the Casting plant.

Besides a recipe administration is integrated. With it is possible to administrate the process parameters of the control of the casting process for various alloys and products easily.

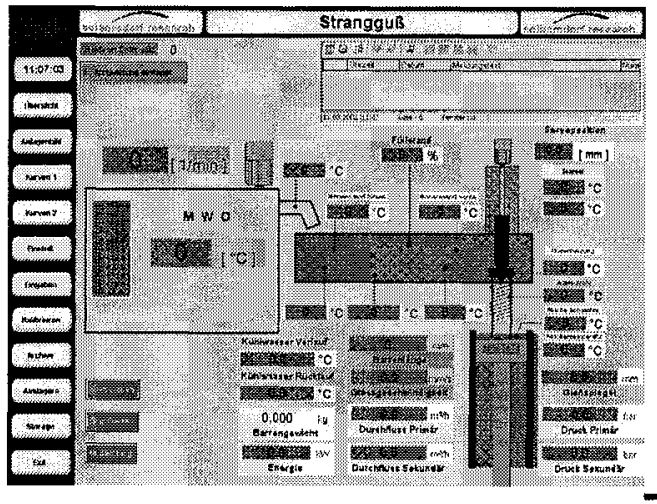

Figure 4 - Visualisation (main panel)

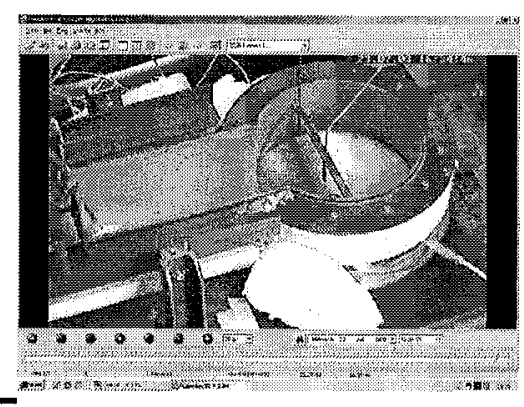

Figure 5 - Live pictures

For a live visualization of the process the control station is equipped with Video monitors and 5 Cameras. They send live pictures directly from the casting pit. These picture a re a lso s hown in the HMI sy stem a nd u sed for online control and visual analysis (Figure 5). 
4.2.3. Telemaintenance via the Internet

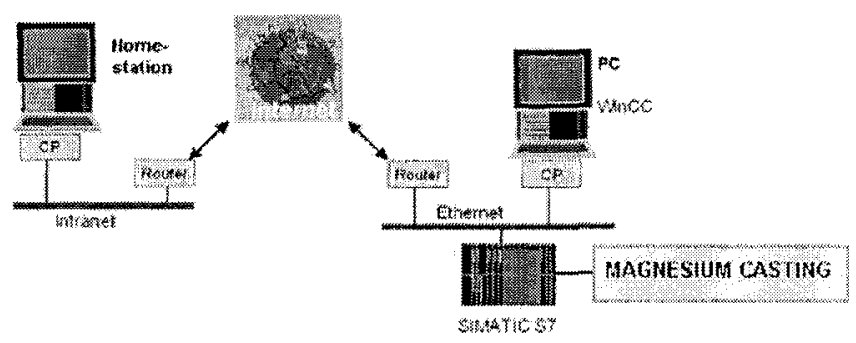

Figure 6 - Telemaintenance via Internet (schematic).

To increase the capacity and improve quality, it's advantageous to connect the magnum-casting machine with the producer. This connection was realisized by Internet (Figure 6). This concept allow to check the status of PLC, report status and alarms, to debug machine diagnostics, to do modifications and enables agreements with customers, to check together material-related machining adjustments and optimisation of production-related parameters from our home station. With this Internet interface it is possible to check these features from any place of the world, providing an Internet connection. Assuming you have the right access privileges to remote control the plant. All drives, sensors, controls and monitoring units are linked to the PC over Ethernet connection and hence these devices can be operated via telematic functional requirements. The communication mechanisms are based on event-driven, high-throughput transfer, resulting in excellent web performance.

\subsection{Control station of a high speed coin sorting machine}

For sorting more than 1000 different types of coins with nearly 100 percent recognition, a unique high-speed coin sorting machine called "Dagobert" was developed by ARC Seibersdorf research $\mathrm{GmbH}$. Based on image recognition, coins in different mintings from approximately 100 countries had to be classified and sorted with a speed of up to 10 coins per second.

\subsubsection{Control System}

The high sorting frequency (10 coins per second) and the time consuming calculations that need to be performed for recognizing each coin require a control system with well-defined communication channels. The control station coordinates all sub-systems. Figure 7 shows the control scheme of the system. In order to maximize system security in service, all relevant data are stored redundantly both in the control station and in the database. Thus sorting data are not lost even if the software crashes.

Via a simple and clearly arranged control terminal, the plant can be controlled conveniently. Any error conditions (synchronization and communication errors, fill level overflow, sensor defects, blockages...) are recognized automatically and - if possible - repaired automatically by special error handling routines. If automatic repair is not feasible, the plant automatically changes to fail-safe operation and allows the user to handle the errors without risking loss of any data. Via the graphical user interface, all sorting parameters (box allocation, allocation to the 
respective benefiting charities, sorting speed, ...) can be adjusted, and all sorting results are monitored.

\subsubsection{Visualisation}

$\mathrm{Via}$ the visualisation at the control station the state of each subsystem can be controlled and monitored. The sorting results are displayed in real-time at the user interface (Figure 8).

The coins delivered to be sorted and counted have been collected and are therefore owned by different charity organizations. For this reason, the sorting results have to be assigned to the respective organization. This assignment is likewise done at the user interface.

\subsubsection{Database}

In order to provide the highest possible data security and to realize real time behaviour, all sorting data of the current batch are stored in the hardware of the control. An Access database is used as front-end for performing data analysis. This database is updated currently with the real time data of the control. Here, the data of all trained coins and their assignments can be monitored and managed.

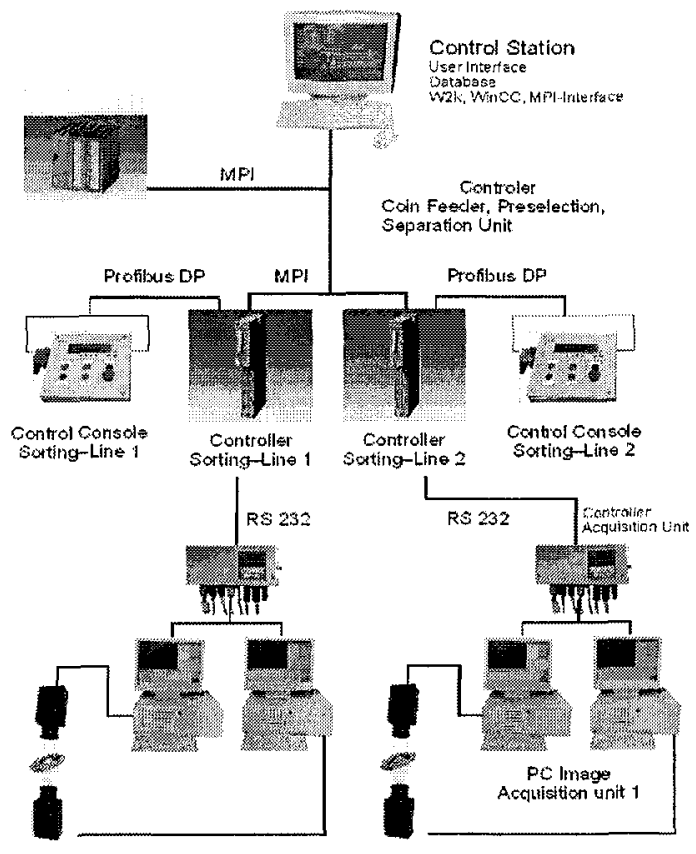

Figure 7 - Control station.
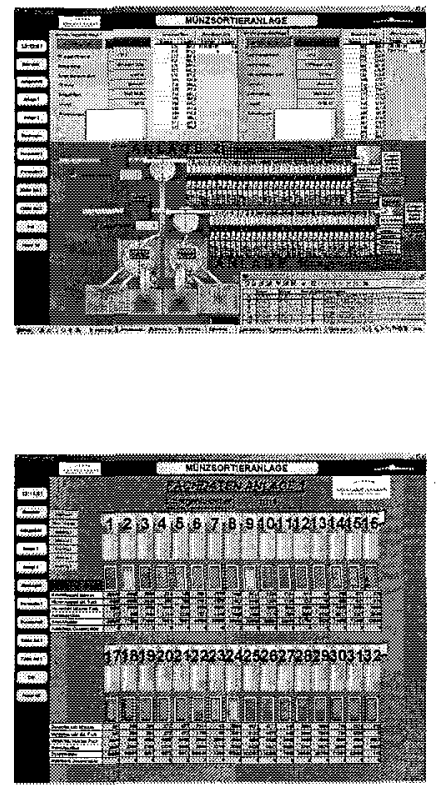

Figure 8 - User Interface

Additionally, a large amount of further information is managed (current exchange rates, processing and delivery state, exchange progress, management of coin containers, ...). A large number of user-definable reports are provided by the database to analyze the entire volume of the collected sorting data (Figure 9).

All data records can be filtered and/or grouped by several parameters (charity organization, processing batch, box number, coin type, currency, ...). At the touch of a button, delivery notes for the respective exchange office can be created. 
Likewise, we can calculate estimates for future results can be calculated any time, based on previously obtained results. This statistical report yields information that is important for organizing future coin deliveries.

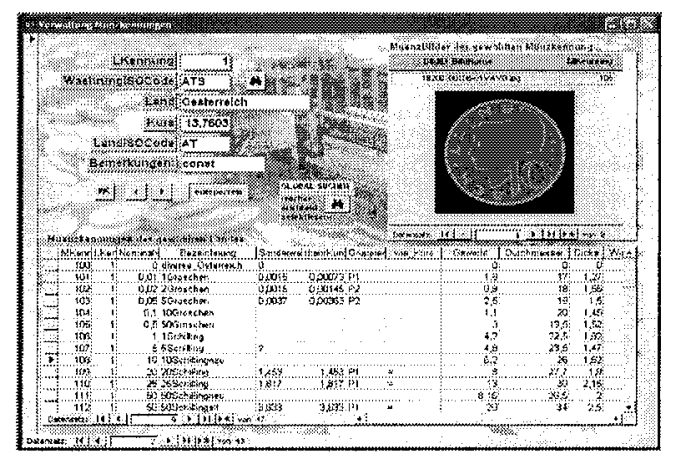

Figure 9 - Coin Administration Tool

\section{CONCLUSION}

Vertical integration of production systems stands for an automated continuous dataflow throughout all enterprise levels. The enterprise network joins enterprise management and production. It optimises resources and productivity, and assures transparent figures in factory management. It controls logistic and production processes, and assures transparent information in production management. It integrates technologies and data, and generates production data in production process.

On the other hand, increasing pressure to enhance productivity, cost-efficiency, competitiveness, and time to market will further drive the demand for ever more sophisticated integration methods and tools.

\section{REFERENCES}

1. A rbeitskreis S ystemaspekte des ZVEI Fachverbandes A UTOMATION, "Die Prozessleittechnik im Spannungsfeld neuer Standards und Technologien" (German), atp Journal 43, pp. 53-60, 2001.

2. OPC Task Force, "OPC Overview", OPC Foundation 1998.

3. "Inside Look at OPC, XML, NET", Start journal, Volume 5, Number 9, 2001.

4. K.C. Laudon, C.G. Traver, "E-Commerce - Business, Technology, Society", Addison-Wesley, 2001.

5. Siemens AG, "Information Security in Industrial Communication", White Paper, 2003.

6. Siemens AG, "Distributed Automation on the Basis of Industrial Ethernet", White Paper, 2000.

7. Dedinak a., Wögerer $\mathrm{Ch}$., "Automatisierung von Großprüfanlagen am Beispiel eines Wärmezählerprüfstandes für die PTB”, (German), White Paper, ARC Seibersdorf research, 2002.

8. Dedinak A., Kronreif G., Wögerer Ch.: "Vertical Integration of Production Systems" IEEE international Conference on "Industrial Technology ICIT'03", Maribor, Dezember 2003.

9. Dedinak A., Koetterl S., Wögerer Ch., Haslinger H.:"Integrated vertical software solutions for industrial used manufacturing and testing systems for research and development", Advanced Manufacturing Technologies - 2004 AMT 2004, London, Ontario, Canada. 